Research Article

\title{
Cross-Modal Travel Route Recommendation Algorithm Based on Internet of Things Awareness
}

\author{
Yuran Zhang ${ }^{1}$ and Ziyan Tang $\mathbb{D}^{2}$ \\ ${ }^{1}$ General Office of Chengdu Municipal CPC Committee, Chengdu, Sichuan 610041, China \\ ${ }^{2}$ Interdisciplinary Center for Culture, Science and Technology, Sichuan University, Chengdu 610065, China \\ Correspondence should be addressed to Ziyan Tang; 20130801@stu.nun.edu.cn
}

Received 25 September 2021; Accepted 16 November 2021; Published 13 December 2021

Academic Editor: Gengxin Sun

Copyright (C) 2021 Yuran Zhang and Ziyan Tang. This is an open access article distributed under the Creative Commons Attribution License, which permits unrestricted use, distribution, and reproduction in any medium, provided the original work is properly cited.

\begin{abstract}
In recent years, the Internet of Things has developed rapidly in people's lives. This brand-new technology is flooding people's lives and widely used in many fields, such as medical field, science and technology field, and industry and agriculture field. As a modern technology, the Internet of Things has many characteristics of low power consumption and multifunction, and it also has the characteristics of data-aware computing. This is the characteristic of this new product. In people's daily life, the Internet of Things is also closely related to people's daily life. In the tourism industry, the Internet of Things can make the best use of everything and give full play to its various advantages as much as possible. The Internet of Things can perceive cross-modal tourism routes. So here, this paper summarizes various algorithms recommended by the Internet of Things for this tourist route and works out the experimental data methods of these algorithms for cross-modal tourism route recommendation. The proposed algorithm is verified by data simulation, compared with related algorithms. We analyze and summarize the simulation results. At present, there is no comparative analysis of the performance of ant colony algorithm, genetic algorithm, and its optimization algorithm in tourism route recommendation. On the basis of crawling the tourism data in the Internet, this paper applies ant colony algorithm, genetic algorithm, max-min optimization ant colony algorithm, and hybrid ant colony algorithm based on greedy solution to tourism route recommendation and evaluates and compares the algorithms from three aspects: average evaluation score, optimal evaluation score, and algorithm time. Experimental results show that the max-min optimization ant colony algorithm and the hybrid ant colony algorithm based on greedy solution can be effectively applied to automated tourist route recommendation.
\end{abstract}

\section{Introduction}

Internet of Things is interwoven by different modern technologies, including wireless communication technology and live data analysis technology, machine language learning technology, sensor technology, and built-in embedded system [1]. The sensors in the internal perception level of the Internet of Things sense all kinds of information about things around them regularly and transmit the information in the perception level to the upper application of the Internet of Things through data fusion and network transmission, thus realizing the Internet of Everything. Therefore, the related research on the key technologies of the Internet of Things based on perception provides a foundation for the development of the Internet of Things [2]. At present, all walks of life at home and abroad have increased the research and development of technologies related to the Internet of Things, which closely links the Internet of Things with life and production. The main application fields of Internet of Things include smart cities, smart homes, intelligent transportation, intelligent environment, and intelligent enterprises. In 2009, IBM Company put forward the innovative concept of "Smart Earth," that is, "Internet plus Internet of Things = Smart Earth" Internet 
of Things detects and perceives object information through the perception layer, which is the Internet of Everything based on perception [3]. As a key technology in the sensing field of Internet of Things, a wireless sensor network is a special ad hoc network [4], which plays a very important role in the perception of Internet of Things. It does not need fixed network support and has the characteristics of fast development and strong survivability [5]. It can carry out remote monitoring and real-time monitoring and can replace people to work in harsh or special environments, such as polluted areas, where the environment cannot be destroyed, and temporary areas, where natural disasters occur. The main applications of wireless sensor networks are as follows: safe construction and monitoring of various large-scale projects; monitoring the environmental conditions of livestock and poultry, crop irrigation, soil air changes and largescale surface detection, and water-saving irrigation control [6]; and marine physics research, pollution monitoring, data acquisition, resource exploration, underwater military target monitoring, positioning, tracking, and classification. Wireless sensor networks in a variety of applications have a common point, either through the location information of nodes in the sensor network to locate nodes or targets or using nodes to track moving objects [7]. With the in-depth application of wireless sensor networks in many fields, especially in the detection and monitoring of enemy bases in military surveillance and rapid location of injured persons in natural disasters, these applications for the need of target location information also make wireless sensor network node location and moving target tracking become the key issues to be solved urgently at this stage [8]. To sum up, the optimization of precision and energy consumption of target location and tracking technology has gradually become a research focus. Target tracking technology has become one of the key technologies of perception-based Internet of Things, which is widely used in military and civilian fields [9]. For the research of target tracking technology, the important factors to be considered are tracking accuracy and tracking energy consumption. At present, there are mature tracking algorithms, and their key research directions include prediction algorithm, tracking structure, and positioning algorithm [10]. At present, in this paper, the ant colony algorithm is used to study the data of cross-modal travel route algorithm under the Internet of Things, and its research direction is to track a single target under the perception of the Internet of Things [11]. The algorithm requires consistent maintenance time, and at the same time, the initial information of all node positions is known. It takes the staying time of the sensor network target as the weight value and adds appropriate calculation to this weight value so as to locate and track more reasonably [12]. The second is a tracking algorithm based on transmission tree, which uses genetic algorithm and optimization algorithm for tracking. The algorithm uses a dynamic tree structure to track dynamically, which is different from the centralized tracking algorithm. The transmission tree is composed of nodes near the target, and the number of nodes increases and decreases dynamically with the movement of the target [13], thus ensuring high-precision tracking, reducing the energy consumption of nodes, and prolonging the network life. In addition, a distributed dynamic cooperative tracking method is proposed, which uses a Gaussian mixture particle filter to estimate the target state [14]. The algorithm uses a Kalman filter to predict linear motion and an extended Kalman filter to predict nonlinear motion, which has high prediction accuracy and uses dynamic cluster structure to track in real time [15]. The algorithm has high tracking accuracy and strong applicability. The shortcoming of this algorithm is that it requires high computing power and storage capacity of sensor nodes [16]. In the diversified modern society, the practical application of multitarget tracking needs to make scholars at home and abroad step up the research and development of target tracking technology in the Internet of Things and thus has achieved many related research results. To sum up, the Internet of Things has great application prospects. The above algorithms are used in other fields for in-depth research, mainly for position tracking and location research. However, in a single algorithm, there is a large error in recommending tourist routes, which makes it impossible to achieve accurate positioning and tracking. The cross-modal model proposed in this paper applies the ant colony algorithm, genetic algorithm, max-min optimization ant colony algorithm, and hybrid ant colony algorithm based on greedy solution to tourist route recommendation and analyzes the average evaluation score and the optimal evaluation score of the selected routes, respectively. This is based on the application of the optimal model in the above research algorithm, which can greatly improve the execution efficiency of the algorithm.

In this paper, a variety of algorithms are studied to combine and optimize the route, and the best algorithm is selected. The path distance error value produced by different tourist attractions has the least influence and can fluctuate in a relatively stable interval value to calculate the optimal path solution, which can greatly reduce the efficiency of travel path selection and travel experience in people's daily life. In the second part of the article, the modeling basis of tourism route algorithm is explained. The third part explains the collection of wireless sensor network routing protocols and related algorithms applied to travel route planning model; the fourth part is the experimental comparison of algorithms combined with various mathematical models.

\section{Overview of Travel Route Algorithm Modeling}

When the traditional travel path engine provides users with search results, regardless of the background of people's travel, returning the same results for different trips does not return personalized results according to people's travel 


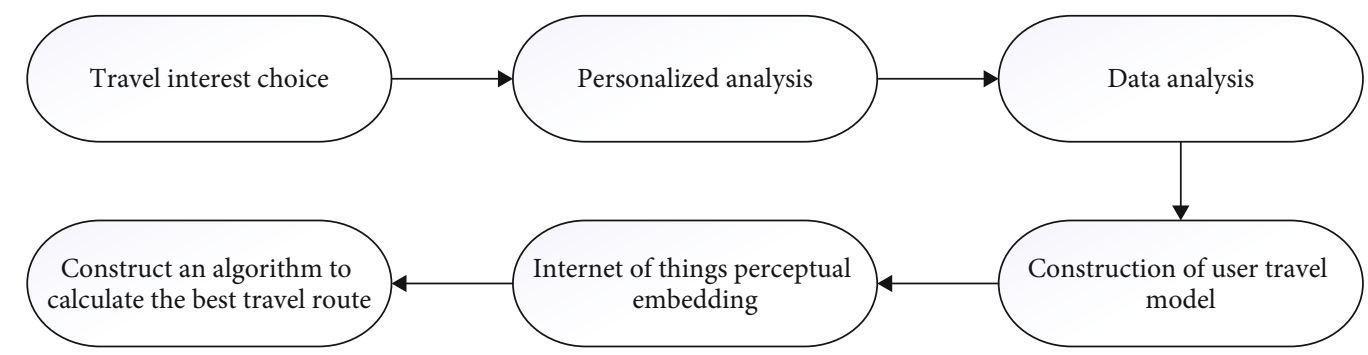

Figure 1: Construction diagram of user travel route modeling algorithm under physical network awareness.

route selection for specific travel modes, so it is necessary to model the route [17], mining people's travel route selection methods by analyzing the historical behavior of travel or the historical browsing content of travel routes. Traveling route modeling refers to the process of establishing the model of data analysis and representation of the same route from the selection of people's travel purpose and mode and the historical information of people's travel behavior, such as the selection mode of people's travel route and the time consumption of travel route. The main purpose is to show the results of selecting the best path for different scenic spots and destinations, so that people can travel quickly and efficiently, and avoid the waste of time caused by people's own path screening [18]. In terms of travel path data modeling, it can be seen from the literal meaning that the established model is based on the travel path, which is closely related to the path distance and time consumption, so it is sometimes called personalized modeling. The result of path selection is not to characterize the optimal path by a literal overview but to get people's optimal path solution by data mining or Internet of Things perception tracking and then express the data obtained from the optimal path solution in a certain data structure format, through the research data analysis of people's path selection for different paths to return targeted results for screening [19]. The second is the data calculation of the algorithm. Through data mining and cross-modal travel path algorithm analysis under the perception of Internet of Things, the collected time error data of various paths are modeled to obtain the calculation of travel path algorithm. This algorithm data calculation is a dynamic process, because the choice of scenic spots determines the length of the path, this data is not static, and the consumption content of different path time will also change [20]. Therefore, the travel path algorithm model also needs to change with the change of path time. As shown in Figure 1, only on the basis of establishing a high-quality travel path algorithm model can the data reliability, rigor, and scientificity obtained from the final optimal path solution be improved to a greater extent.

Choose the corresponding interest route for travel and plan the corresponding route. Enter the individual analysis, and recommend some interesting routes according to personal interest points. According to the above personalized data, the travel route of users is constructed. After in-depth analysis of the information such as people flow and road conditions perceived by the Internet of Things, the best travel route for users is finally constructed.

\section{Routing Protocol for Energy Acquisition in Wireless Sensor Networks}

A traditional Internet of Things routing algorithm has been quite mature, but for the energy acquisition of wireless sensor networks, routing algorithm research is not particularly in-depth [21]. At present, there have been some research results of routing algorithms with perceptual performance, and the existing research is mainly planar routing algorithm protocol. The invention relates to a routing protocol with a special energy collection mode, which can take the data energy collection work as a special consideration factor of an energy-saving routing protocol. The routing algorithm first proposes a hybrid routing metric which combines the residual energy and energy collection rate of nodes and then proposes a mechanism to update the information of neighbor nodes. According to this mechanism, the information of neighbor nodes is updated [22]. Nodes can choose the optimal data down-transform sampling location autonomously, considering the correlation between the hybrid routing metrics and the amount of node information in the adjacent location area. At present, an opportunistic routing protocol specially used for the best adaptation of Internet of Things awareness is obtained. Routing divides nodes into groups and determines the transmission priority of each node by considering the available energy of each node and the distance from the node to the sink node [23]. Simulation results show that adaptive opportunistic routing has better throughput, fairness, and scalability than other routes. Geographical routing is added to the routing of energy acquisition wireless sensor networks, and the routing is considered by geographical location, which makes the energy acquisition nodes get better performance [24]. Using the idea of geographic routing, we continue to optimize this route and get a geographic routing algorithm with repeatability detection; that is, we introduce a repeatability detection mechanism on the basis of geographic routing. Before forwarding data packets, we check whether the same data packets have been sent before, and if so, we discard the current data packets, thus improving network performance and network forwarding efficiency [25]. Different layouts and application scenarios of energy collection nodes are analyzed, different routing protocols are compared and analyzed, and the routing algorithm and node location indication suitable for the optimal path solution algorithm are found out. A cooperative path-aware algorithm for throughput optimization based on path algorithm model is 


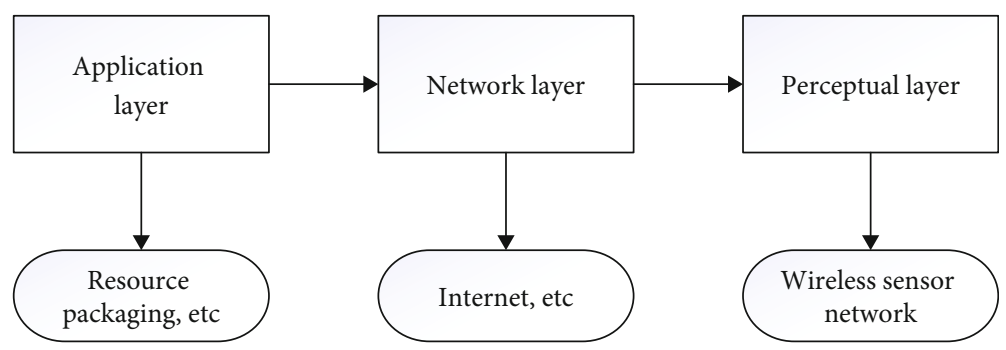

FIGURE 2: Construction diagram of Internet of Things awareness module.

proposed. These algorithms show effective improvement and performance improvement for power scheduling problems with different time delays. The existing routing algorithms for IoT awareness are mainly planar, while the traditional hierarchical routing algorithms for IoT applications are mainly aimed at isomorphic sensor networks; that is, the initial energy of each node is the same [26]. The nodes in the data acquisition wireless sensor network are heterogeneous; that is, the energy is different. If we use the classical clustering routing protocol in IoT awareness, the nodes with less energy will sleep or die because of excessive energy consumption, which will lead to network failure. Therefore, the traditional clustering routing algorithm in IoT awareness cannot be directly applied to the optimal path algorithm model. The structure diagram of each layer of the current Internet of Things is shown in Figure 2.

3.1. Solve the Mathematical Model of Tourism Route Planning. In order to compare the mathematical models based on the basic genetic algorithm of tourism route, in this paper, the central idea and architecture of hybrid ant colony algorithm are proposed. And the hybrid ant colony algorithm is used to recommend the best route for tourism. The main architecture of some mathematical algorithm models involved includes chromosome self-coding, fitness function selection, and data analysis operator architecture. The detailed mathematical evaluation methods and algorithms of these algorithm models are described as follows:

(1) Chromosome coding: people's common travel routes can be displayed by binary coding of machine language. For example, chromosome sequences in the number 10 dimension can be divided into $(0,1,0$, $1,0,0,0,0,0,1)$ to represent, and then 2,4 , and 10 are selected into the current best routes according to chromosome coding characteristics.

(2) Fitness function: in the general genetic algorithm, the key and most important thing is the construction mode of fitness function architecture model. At present, it is used to evaluate and select the target chromosome data source and can calculate the corresponding data genetic probability interval which affects the single target in the framework of selection operator, which is the key factor index reference of whether the popular genetic algorithm can achieve the optimal solution path. Therefore, the selection of appropriate fitness function can directly affect the convergence and occurrence speed of genetic algorithm and whether it is enough to find the optimal path solution function. The mathematical evaluation model of appropriate function constructed in this paper is as follows:

$$
\begin{gathered}
\text { fit }(f(x), g(x))=(1-\partial)\left(\frac{A_{\max }}{1+A_{\min }+f_{(x)}}\right)^{y}+(g(x))^{\partial}, \\
\text { pis }=\frac{F_{i}}{\sum_{i-1}^{M} F_{i}} \quad(i=1,2, \cdots, M), \\
\sum_{k=1}^{m} \sum_{j-1}^{n-1} x_{o j k}-\sum_{k=1}^{m} \sum_{i=1}^{n-1} x_{i o k}=0, \quad \forall k \in A,
\end{gathered}
$$

where fit $(f(x), g(x))$ is the fitness of tourism classic $x ; f(x)$ is the objective optimization function of the scenic spot cost, which is composed of information such as scenic spot ticket price; $g(x)$ is the target optimization function of the scenic spot heat, which represents the total number of visitors to the scenic spot; $A_{\min }$ is the minimum cost and $A_{\max }$ is the maximum cost; and gamma is the scaling factor of cost and the scaling factor of heat. The function of Gamma is to prevent the value of cost or heat from fluctuating too much, in order to balance the influence of cost and heat on moderation.

(3) Select operator: using roulette, firstly, the sum of fitness corresponding to all populations is calculated; then, the relative fitness $\mathrm{fit}_{i} / \mathrm{sum}$ of each individual is calculated as the probability of roulette, each probability value forms an area, and the sum of all probabilities is 1 . Finally, a random value between 0 and 1 is generated, and the number of times each individual is selected and determined according to the occurrence of the random number in the above probability area. And adopt the elite rule, and forcibly copy the best individual of the previous generation directly into the next generation, so that the individual of the next generation will be superior to the previous generation. $U_{\max }$ and $U_{\min }$ are the maximum and minimum dependent variables, respectively. 


$$
\begin{gathered}
p_{i}=p_{i-1}+\frac{\mathrm{fit}_{i}}{\text { sum }} \\
\text { sum }=\sum_{i=1}^{n} \mathrm{fit}, \\
\delta=\frac{U_{\text {max }}-U_{\min }}{2^{l}-1}, \\
x=U_{\min }+\left(\sum_{i=1}^{l} b_{i} \times 2^{i-1}\right) \times \frac{U_{\max }-U_{\min }}{2^{l}-1} .
\end{gathered}
$$

(4) Genetic algorithm: a genetic algorithm is completely not used for other external comprehensive information in the process of systematic search and selfevolution. It only calculates the advantages and disadvantages of individual solutions through the evaluation of functions and uses this as the fundamental basis for the operation of subsequent genetic steps. Because of the construction of genetic algorithm and fitness function to compare permutation number and on this basis to calculate the size of the probability of the selection of data, so the fitness function of the selection of the value of the integer value should be selected. Therefore, in many cases, fitness function is especially necessary if the objective function is demapped to the traditional form of finding the maximum function value and the randomly selected function value is nonnegative:

$$
\begin{gathered}
f(x)=x \cdot \sin (10 \cdot \pi \cdot x)+2, \\
p_{i}=\frac{f\left(x_{i}\right)}{\sum_{j=1}^{n} f\left(x_{i}\right)}, \\
\left(b_{0}, \cdots, b_{20} b_{21}\right)_{2}=\left(\sum_{i=0}^{21} b_{i} \cdot 2^{i}\right)_{10}=x^{t} .
\end{gathered}
$$

(5) Ant colony algorithm: literally speaking, it is equivalent to ants finding the shortest path, which is due to pheromones and environment. Assuming that there are two paths for ants to choose from the food point to return to the ant nest, the number of ants on the first two paths is equal. When an ant reaches the target point, it will immediately return according to the original path. The ants on the path with relatively short distance in the two paths consume a short time to go back and forth, and the frequency of repetition increases. The number of ants going back and forth in a certain period of time will increase, and the amount of information left in the path will also increase, which attracts other ants and leaves more information than before. On the other hand, the relatively long path will be the opposite, so more and more ants will leave information back and forth to reach the shortest path. In the data, $T_{t}$ represents the path variable, $n$ represents the dependent variable, $t$ represents the distance independent variable, and $P_{u}$ represents the number of ant independent variables, so the following ant colony algorithm formulas can be obtained:

$$
\begin{gathered}
T(t)=(1-p) \cdot T_{u}(t-1)+\Delta T_{u}, \\
\Delta T_{u}=\sum_{k=1}^{m} \Delta T_{u}^{k}+\lambda \Delta T_{u}^{b}, \\
p_{u}^{k}=\frac{T_{n}^{\alpha} \partial_{u}^{\beta}}{\sum_{j} T_{u}^{\alpha} \partial_{u}^{\beta}}, \\
T_{u}(n+1)=p \times T_{u}(n)+\sum_{k=1}^{m} \Delta T_{u}^{k} .
\end{gathered}
$$

(6) Hybrid ant colony algorithm: the corresponding model formula is constructed by greedy solution combined with the ant colony algorithm. On the basis of ant colony algorithm, increase the number of matrix columns. The information amount structure is added. Therefore, the dependent variable will have more reference value intervals. Through the number in the interval, the error of the final calculation value will be smaller. Comparison based on the travel route recommendation algorithm can form a comparative data format, and the optimal solution can be obtained by studying the error interval of the data volume in combination with the perception of the Internet of Things. Therefore, the formula of the hybrid ant colony algorithm model is as follows:

$$
\begin{gathered}
m=\sum_{k=2}^{n} b_{i}(t)(n \in k), \\
\Delta T_{i j}^{k}=\left(1-p_{1}\right) \cdot T_{i j}(t)+p_{1} \frac{1}{L_{\max }}, \\
V_{i+1}=w V_{i}+c_{1} r_{1}\left(P_{a}-X_{i}\right)+c_{2} r_{2}\left(G_{1}-X_{i}\right), \\
\sum_{k=1}^{m} \sum_{j=1}^{n-1} x_{0 j k} \leq m, \\
\sum_{k=1}^{m} \sum_{i=0}^{n-1} x_{i j k}=1, \quad \forall v_{j} \in C, \\
\sum_{i=1}^{n-1} q_{i} \sum_{j=0}^{n-1} x_{i j k} \leq G, \quad \forall k \in A .
\end{gathered}
$$

In the comprehensive algorithm, $V_{i+1}$ is the optimal solution of data amount, $P_{1}$ is the distance variable, $L$ is the distance return value, $c_{1} r_{1}$ is the first product value of 
the return value, $c_{2} r_{2}$ is the second product value, and $T$ is the termination variable. In order to select the best tourist route, an evaluation model is established to evaluate whether it is the best tourist route. The idea of modeling is that the selected tourist routes give priority to popular scenic spots, and the sum of scenic spot ticket price and hotel expenses is low. Therefore, an optimal score function of tourist routes is defined, which is used to evaluate the score values of tourist routes obtained by different algorithms, and a high score indicates that the route is excellent.

$$
\begin{gathered}
u_{t}=\frac{E_{1} \cdot R_{t}+E_{2}(k) \cdot R_{k}(t)}{N_{c} \cdot\left(E_{x}+E_{1}\right)}, \\
N_{c}=1+\sum_{a \in c} N^{a} x^{a}, \\
X_{C}=\partial X_{c}\left(u_{c}-u^{a}(x)\right), \\
u^{a}(x)=\sum_{C_{1}} x_{c} \cdot u_{c}^{a}, \\
E_{e h}=R_{i}(t) \cdot \Delta t, \\
R_{i}(t)=a_{i} e^{\left(t-b_{i}\right)^{2} / 2 c_{i}^{2},}
\end{gathered}
$$

where $R_{i}$ is a variable frequency of wireless sensor in Internet of Things. Through the location and tracking of variable perception by frequency conversion, it can make the travel route more localizable. $N_{c}$ is the network layer coefficient value of the Internet of Things. When the coefficient layer of the Internet of Things changes, the discreteness can become uncertain, and the calculation error becomes larger due to the influence of fluctuation. $X_{c}$ is the wireless coefficient of independent variable, and the wireless coefficient increases with the increase of induction times. Finally, the cross-modal perception error value of $u_{t}$ can be obtained through mathematical evaluation model calculation.

3.2. Selection and Analysis of Experimental Data of Travel Route. Scholars at home and abroad mainly focus on improving the ant colony algorithm; however, when the improved ant colony algorithm is efficient or can achieve their expected results, this often needs to be verified in examples. This chapter gives the comparison of the data before and after the algorithm is improved in the form of examples. When there are more data, the idealized data in the standard database is used. Some scholars use the given point coordinates when studying the route planning of vehicle routing and then use the given point coordinates to get the straight line distance between two points and use the obtained distance instead of the distance value in the actual road condition, which is obviously inconsistent with the actual road condition. What we can get from the current research data is that suppose a tourist enthusiast is going on a go on road trip and after traveling to $1074 \mathrm{~A}$-level national key tourist attractions, these 107 tourist attractions are located in different provinces and places. In this way, the independent variable and dependent variable data of tourist route will be affected by the number of tours in different years and the number of days from departure to completion of a tour. The total expenditure of tourism, travel time, travel choice mode, travel time, and so on are constrained by external conditions, which can be summarized into discrete values to express this uncertain factor. Once again, the cost of travel can include many factors to constitute other expenses, such as the cost of expenses on the way, the toll of the road, and the accommodation expenses along the way. It is assumed that the mode of transportation for the whole journey is self-driving. If one or several factors are regarded as invariants, other factors can be determined, a mathematical model can be established, and the optimal route can be planned under the corresponding constraints. Because these 107 scenic spots belong to different provinces, therefore, we give priority to various scenic spots in the province as independent variables. Then, the shortest time ratio of each scenic spot in each different province is calculated. Then, the mathematical evaluation model under the perception of Internet of Things is established in turn to study. Then, the best travel path chart mode of different scenic spots in the same province is planned, and the big data perceived by the same Internet of Things finds out the distance between different scenic spots in the province. According to the planned road map, the time required from the unit scenic spot to the next unit scenic spot can be studied in turn. Moreover, the total travel time of scenic spots in each province and the total time required for a unit scenic spot to another unit scenic spot are the total travel time consumption data in each same province. Then, the provinces were taken as the unit, and the mathematical algorithm evaluation model is established in turn. Under the control of corresponding constraint indexes, the best road map between provinces is planned, and the time needed from one province to another is calculated. The total time loss of each trip is the total time needed from the target province to the unit province and the total time consumption data of playing in the province. As shown in Figure 3, based on the data selection and construction of people's travel routes, on the one hand, random sampling is adopted for the number and time of routes of each scenic spot, and on the other hand, random sampling is adopted for data selection to ensure the comparability and rigor of data. For sampling, it is necessary to ensure that batch calculation can be carried out in the research of path analysis and calculation of several algorithms, and wireless sensing embedded analysis data can be carried out for travel under the perception of Internet of Things. Combined with algorithms, uncertainty can be reduced and dimension interval can be controlled. Therefore, it is necessary to grasp the data selection of scenic spots. Recently, the tourism department published a report on China's tourism development. According to the data, there are $2015 \mathrm{~A}-$ level scenic spots in China. In the past two years, the growth rate decreased by $6.29 \%$ and $8.06 \%$, respectively. The results show that the development of $5 \mathrm{~A}-$ level scenic spots has entered a stable period, and the advantages of 5A-level scenic spots in tickets show a downward trend. By the second half of 2014, the peak season tickets of 5A scenic spots decreased by 22.15 percentage points and 1.94 percentage points, respectively, compared with 2018 and 2019, with an average ticket price of 103 yuan/ticket and an average off- 


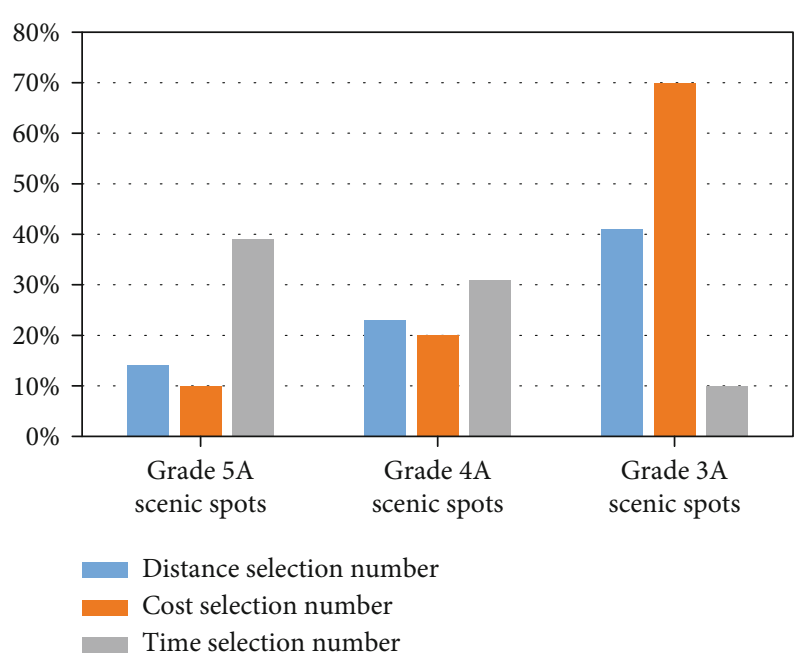

FIGURE 3: Histogram of selection of tourist route attraction data.

season ticket price of 93 yuan/ticket. Compared with that in 2019 , the total price decreased by 19.88 percentage points as a whole, and for 2013 , it decreased by 1.33 percentage points. In 2020, 186 5A scenic spots were searched for keywords, and the network attention of $5 \mathrm{~A}$ scenic spots increased significantly compared with 13 years, with an increase of $21 \%$. This research report counts the daily average of Baidu Index, and uses Baidu Index Search Platform to search the results, which reflects the network attention of $5 \mathrm{~A}$ scenic spots in 2014.

Distance, cost, and time are important indicators for users to measure travel itinerary, so it is great research significance to choose these three indicators for comprehensive comparison. As can be seen from Figure 3, a scenic spot has an advantage in cost and low price, but the travel time is short and the user experience is not ideal. $4 \mathrm{~A}$ scenic spot is the experience standard between $3 \mathrm{~A}$ scenic spot and $5 \mathrm{~A}$ scenic spot. According to the individual needs of customers, the corresponding concerns in these three indicators are selected, and the corresponding scenic spots are appropriately recommended, which has an efficient recommendation rate.

\section{Experimental}

For the recommendation experiment of tourism route recommendation algorithm based on Internet of Things awareness, in this paper, several randomly selected variable data such as route time, distance, and cost of scenic spots are applied to the algorithm model. Calculate the selected data to obtain the final data with the shortest path and the data with the least time consumption, modeling, and applying the algorithm; using the sensing technology of Internet of Things to track the path and time, this paper uses four algorithms to apply calculation to different scenic spot travel data, which are the ant colony algorithm, greedy algorithm, hybrid ant colony algorithm, and genetic algorithm. At present, these four algorithms have been partially studied, which are convenient for the construction and calculation of the algorithms. They are modern computing methods, which can effectively combine the Internet of Things sensing technology, and the tracking delay represents the time difference between the time when the location information is obtained by tracking and positioning and the specified time. The simulation start time of the three algorithms is the same, and the tracking delay is obtained by the average value of the difference between the determined time and the actual set time of the position information obtained by several tracking algorithms in the actual trajectory. The tracking simulation of networks with different numbers of nodes is carried out, and the trend of tracking delay with the number of nodes in the network is obtained. Sensor tracking and positioning is a simple and effective positioning method, which is suitable for a home environment. By judging the position, it can judge whether the user is located in the signal blind area and both sides of the wall and then give priority to the network access problem of users in this area. Tracking and positioning of indoor moving targets mainly include two steps: target position prediction and target positioning. Aiming at the characteristics of simple motion and low speed of moving targets and weak computing power of sensor nodes in smart homes, the dynamic cluster structure is used to track, the prediction algorithm adopts grey model based on wavelet denoising, and the trilateral positioning method based on RSSL is used to realize coordinate positioning.

4.1. Comparison of Experimental Data. The data comparison diagram of the four algorithms for the same path is shown in Figure 4. It can be seen that the ant colony algorithm can effectively infer the shortest path for scenic spots with short relative distance, and among the four algorithms, the ant colony algorithm is not particularly prominent in calculating the time of travel route. On the contrary, hybrid ant colony computing can greatly improve and analyze data on this basis. Under the tracking based on Internet of Things perception, the performance of the algorithm is evaluated by tracking accuracy, which is measured by the distance between the tracking position and the actual target moving position, and the average accuracy after 100 seconds of movement is taken. And compare the size of tracking structure. After 10 rounds of motion, the tracking accuracy map is obtained. Different curtain structure sizes affect tracking accuracy. The tracking distance radius is $1200,1000,800$, and 600 meters, respectively, and the corresponding tracking accuracy is lower than 0.4 meters. The smaller the radius of dynamic cluster structure, the worse the tracking effect. When the cluster structure becomes smaller, it contains fewer tracking nodes, which leads to the reduction of tracking information and the decline of dynamic cluster tracking accuracy.

The accuracy of ant colony algorithm to path, the error rate of the calculated data of the average optimal path, and the theoretical optimal path under the change of discrete values are shown in Figure 5. It can be seen that the ant colony algorithm has values between 0.2 and 0.5 , so some approximate discrete values between 0.2 and 0.5 can be obtained after synthesis, which are $0.2,0.3,0.4$, and 0.5 , respectively. The influence of the change of discrete value 


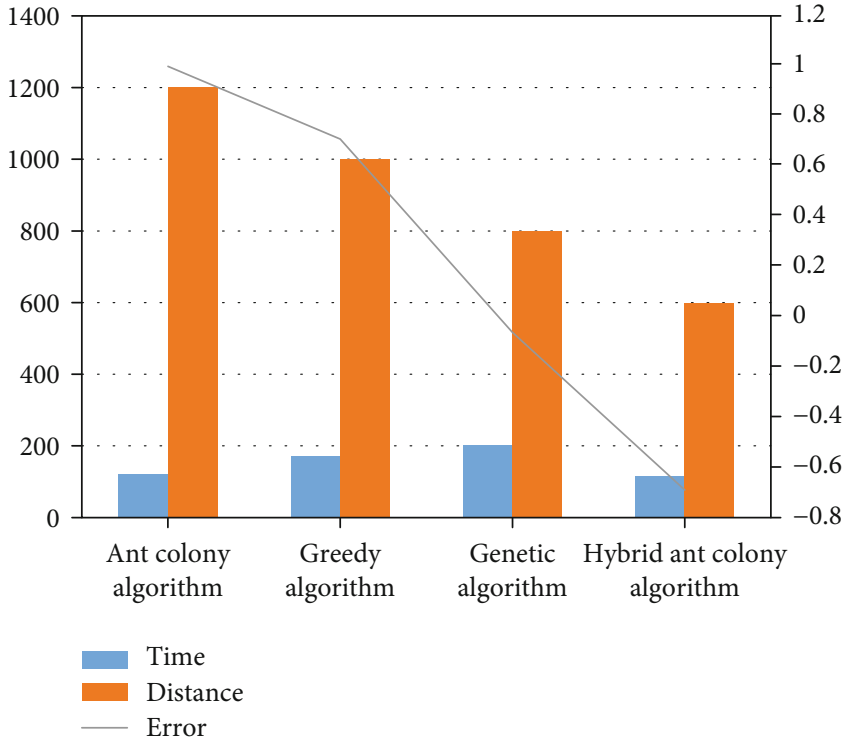

FIGURE 4: Comparison of time and distance data of four algorithms.

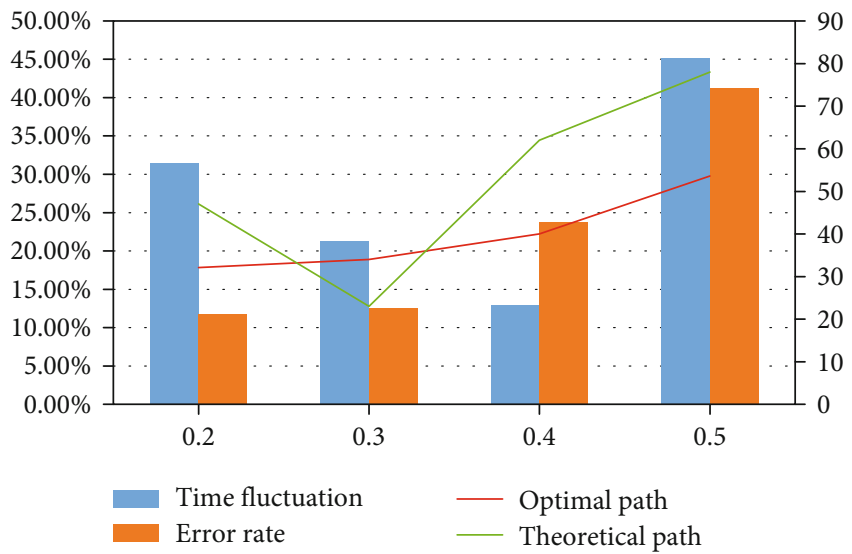

Figure 5: Influence of discrete numerical values on ant colony algorithm data comparison chart.

parameters on the operation results is expressed. When the discrete value is 0.2 , the calculation error rate of ant colony algorithm is always maintained in a normal interval domain. Although the error value increases when the discrete value is 0.3 , it still tends to a normal interval level. However, when the discrete value reaches 0.4 , the error value gradually increases beyond the normal interval area, and when the discrete value reaches 0.5 , the error value reaches its peak, which exceeds the normal interval a lot. It can be seen that for the ant colony algorithm, the influence of discrete values perceived by the Internet of Things on the calculation error rate of ant colony algorithm is not significant when the fluctuation is 0.2 and 0.3 , but when it exceeds 0.3 , the influence on the error rate of ant colony algorithm presents a positive increasing trend. Therefore, the comparison in the experiment should control the variable selection of discrete values.

When the constraint condition is invariant, the genetic algorithm uses multiple regression calculation and iterative calculation to calculate and analyze the path, so the error

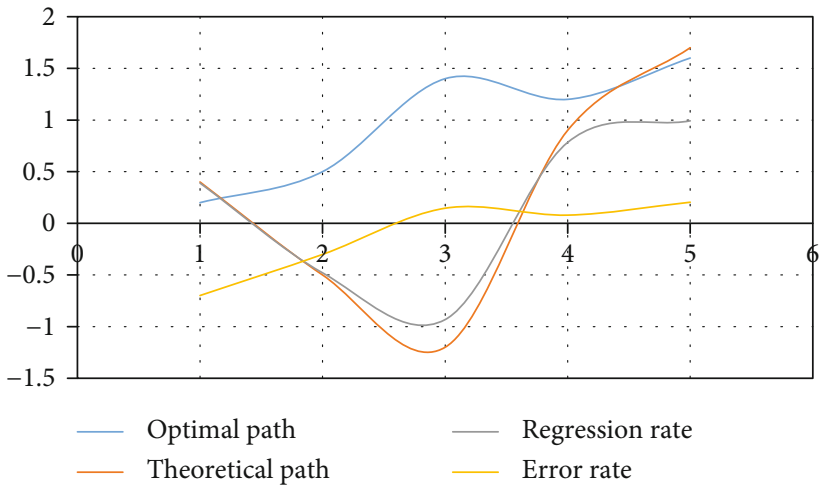

FIgURE 6: Comparison of error data in time selection by the genetic algorithm.
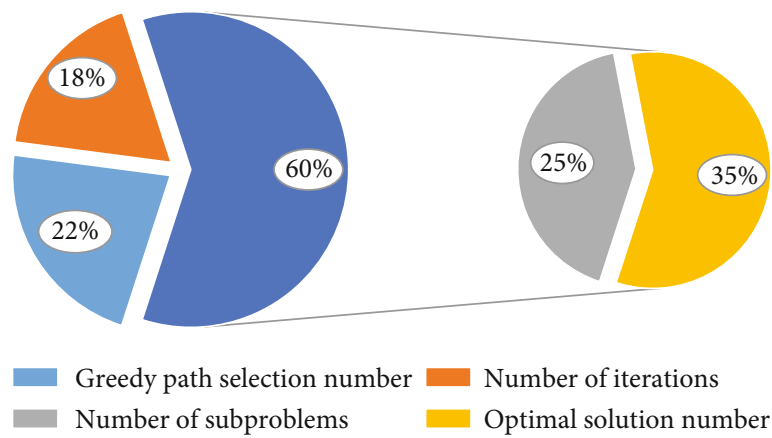

FIgURE 7: Data comparison graph between greedy path selection number and optimal solution of greedy algorithm.

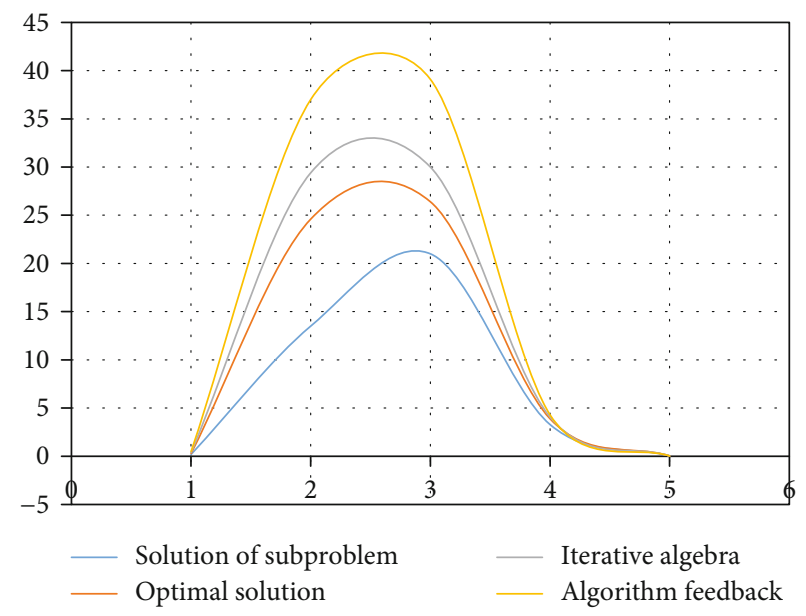

Figure 8: Data graph of the influence of greedy algorithm time scale on the optimal solution of subproblem.

value is not so obvious. But in terms of time calculation, the ability of genetic algorithm to analyze data produces a deviation value. Therefore, in the planning of travel routes, the selection of time parameters will have corresponding influence on the data of genetic algorithm. As far as the genetic algorithm is concerned, the selection of different time parameters can be seen from the data analysis diagram in Figure 6. When the time takes the normal interval number, the analysis and calculation of path by the genetic 


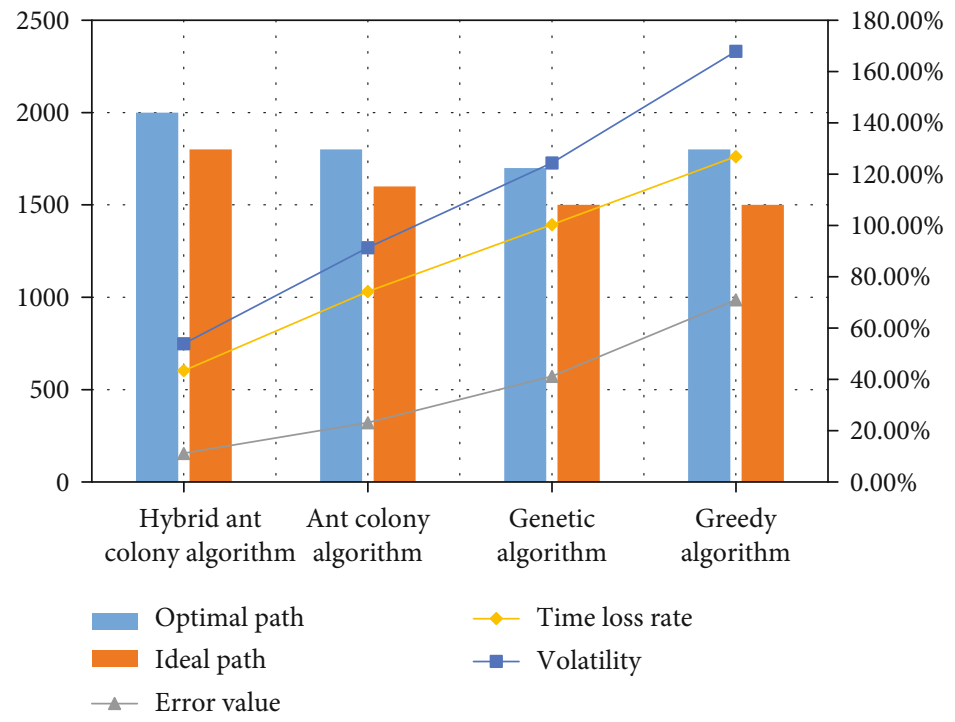

FIGURE 9: Comparison chart of experimental data of various algorithms.

algorithm are that there is no obvious fluctuation in a relatively normal area, and when the time is above 4 scales, the calculation data of the path gradually produces the deviation from the optimal path data, showing an increasing trend of proportional function. Therefore, when calculating the travel path by genetic algorithm, the time parameters should be selected in the positive range. When the unit time is selected to exceed 4.32 , the deviation of the path reaches $41.2 \%$, while the normal interval should fluctuate in the interval of $23 \%$ $30 \%$. The results show that the optimal solution obtained by the genetic algorithm is within a certain error range with the known optimal solution; it can find the path sequence and path value which are very close to the known optimal solution provided by the database. The solution accuracy of ant colony algorithm in this paper is very close to the known optimal solution at present, which reflects the rationality of the genetic algorithm designed in this paper to solve the route from the side, and has reference significance.

In the greedy algorithm, the display form of data is more accurate, and the greedy algorithm is a kind of algorithm design theory that calculates the optimal data of some data more simply and quickly. Generally speaking, the biggest feature of greedy algorithm as travel path analysis is to calculate step by step. Usually, based on the current state as the theoretical basis, we seek an optimal test as the best selection of path data, but from this aspect, we abandon some data comparison of external conditions, which saves a lot of unnecessary time consumption and data calculation for seeking the optimal solution data. The greedy algorithm is different from other shortest path algorithms. It adopts a top-down calculation method. Through iterative model construction, the inherited greedy selection data is selected, so every time greedy data selection is carried out, and all complex problems will be reduced to a small sub-problem data to deal with, so as long as each step of greedy selection is carried out, the greedy data solution of a subproblem can be obtained. Although greedy data of subproblem can be obtained in every step, the unique solution of all problems cannot be guaranteed to be optimal data sometimes, so the greedy algorithm cannot take the way of data backtracking. As shown in Figure 7, it can be seen that the influence of greedy algorithm on the number of iterations and the number of subproblems fluctuates in the comparison data of the number of greedy path selections.

From the data obtained from the solution of the greedy algorithm to the optimal travel path, it can be seen that the influence of the fluctuation of discrete values on the greedy algorithm lies in the fact that the lower the discrete values, the greater the fluctuation. This is a reverse trend compared with the genetic algorithm. In terms of time consumption, a discrete value has little influence on the greedy algorithm, but in terms of error, when the discrete value is selected as 0.2 , the volatility reaches its peak and exceeds the normal value interval, when the discrete value is selected as 0.3 , the volatility gradually decreases, and when the discrete value is selected as 0.5 , the error value is the smallest and the volatility is relatively stable. The calculated optimal path has the shortest solution distance and the least iteration times. The greedy algorithm based on Internet of Things perception calculates data pairs for travel paths, as shown in Figure 8.

In the greedy algorithm for the optimal solution of the subproblem, the subproblem solution, the optimal solution, iterative algebra, and algorithm feedback are analyzed. The optimal solution, iterative algebra, and algorithm feedback show the best performance at 2.5 times, while the subproblem solution shows better performance at 3 times. The whole algorithm shows the best performance from 2.5 to 3 times.

The hybrid ant colony algorithm is also called ant colony optimization algorithm. On the basis of ant colony algorithm, information source elements are used to construct information mutually. The optimization algorithm constructed by information particle swarm optimization can make use of the position information of single individual at present. The current iterative algorithm calculates the three information sources, namely, the limit value area of the single unit before calculation and the limit value area 


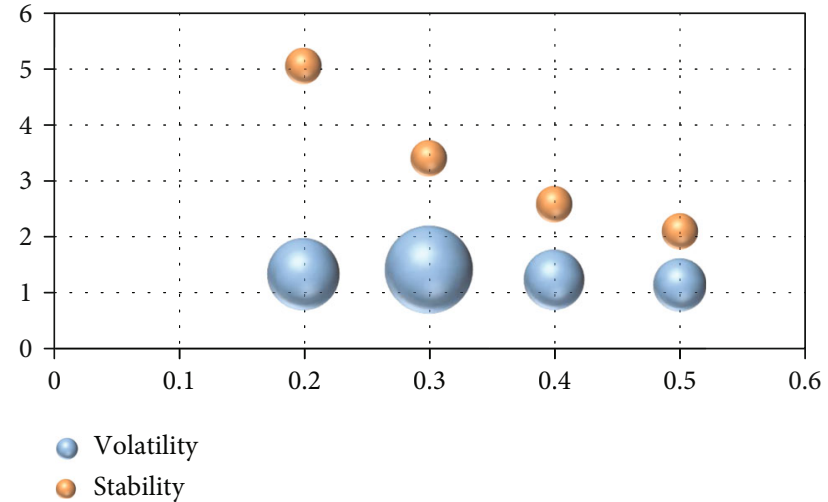

FIgure 10: Comparison chart of volatility and stability data of hybrid ant colony algorithm.

constructed globally. Through the change of speed, the coverage and compilation of the position data of the single unit can be completed, and then the improvement measures can be produced for solving the optimal data of the path problem. According to the current experimental research, the above common ant colony algorithm is also particularly prone to resulting data and falling into the analysis of local optimal solution data. And the data solved by ant colony algorithm is not particularly fast. Therefore, the hybrid combination algorithm of common ant colony algorithm and data particle swarm optimization algorithm can be used, which is called the hybrid ant colony algorithm. It can complement the deficiencies in the path algorithm, thereby improving the data generation speed of the optimal solution of the algorithm and solving the problem of local optimum for a single problem path. It can be constructed according to the hybrid algorithm model of common ant colony algorithm and data particle swarm optimization algorithm. After the algorithm flow completes an overall calculation according to the normal ant colony algorithm, then, we let ants adjust and analyze their positions according to the individual optimal limit range in particle swarm optimization and the optimal problem data value of the whole path problem; then, this hybrid algorithm can be applied to the calculation of traveling salesman path problem at present. As shown in Figures 9 and 10, it can be seen that the hybrid ant colony algorithm has obviously improved the time of travel path analysis and the calculation of the optimal path. And the optimal path can be quickly obtained in a short time and reduce the time loss rate. When the discrete value is selected, it will not receive the fluctuation change, so that the data of the optimal solution of the path obtained by the algorithm will have an absolutely stable trend. In the theoretical path, the hybrid ant colony algorithm can also achieve the analysis of the target exponential rate and the overall calculation of the data, so that the distance of the travel path can be optimized.

\section{Conclusion}

Carrying the ant colony algorithm, genetic algorithm, greedy algorithm, and hybrid ant colony algorithm in the research of travel path data combined with Internet of Things sensing, by analyzing the theoretical path solution of these algorithms to the optimal path solution and time loss, according to the comparison of several data, such as the error of discrete value numerical selection analysis, the hybrid ant colony algorithm is an algorithm model recommended by cross-modal travel path comparison based on Internet of Things perception. Based on this algorithm model, the influence of path distance error value produced by different tourist attractions selection is minimized. The optimal path solution can be calculated by fluctuating in a relatively stable interval value. This can greatly reduce the efficiency of choosing travel routes and the travel experience in people's daily life. Under the perception of today's Internet of Things, although the other three algorithms have their own advantages and disadvantages, the path selection can be made in a short time, but the discrete values are 0.2. The error of the selection of $0.3,0.4$, and 0.5 is $18.7 \%$ beyond the normal value range. In the optimal path, the value produced by the fluctuation of iteration times of greedy algorithm is also in the fluctuation range of $42.3 \% \sim 47.1 \%$, and the time loss rate produced by the ant colony algorithm is repeated back and forth in the range of $13 \% \sim 18.2 \%$. But the hybrid ant colony algorithm solves this problem. In the selection of discrete values, the error value can also be kept fluctuating in the normal range of $10 \% \sim 15 \%$. And it can greatly reduce the peak data of about 17.32 in terms of time loss. In terms of regression value, the regression value of hybrid ant colony algorithm (3.971) is far lower than that of ant colony algorithm (0.23). When it is negative, the choice of optimal path also produces a negative interval domain of $-1.172 \sim 1.42$. Therefore, the construction and calculation of hybrid ant colony algorithm are recommended in the research of cross-modal travel path algorithm based on Internet of Things awareness. A future work focuses on multiroute optimization in the application of various algorithms and may consider the road conditions, traffic, and people flow of the routes for comprehensive application. According to the current application of tourism websites, this paper comprehensively evaluates the hot spots and whether to recommend the corresponding routes.

\section{Data Availability}

The experimental data used to support the findings of this study are available from the corresponding author upon request.

\section{Conflicts of Interest}

The authors declared that they have no conflicts of interest regarding this work.

\section{References}

[1] P. C. Garrido, G. M. Miraz, I. L. Ruiz, and M. Á. Gómez-Nieto, "A model for the development of NFC context-awareness applications on internet of things," in 2010 Second International Workshop on Near Field Communication, pp. 9-14, Monaco, Monaco, 2010. 
[2] L. Lim, P. Marie, D. Conan, S. Chabridon, T. Desprats, and A. Manzoor, "Enhancing context data distribution for the internet of things using qoc-awareness and attribute-based access control," Annals of Telecommunications, vol. 71, no. 34, pp. 121-132, 2016.

[3] M. Vazquez-Briseno, C. Navarro-Cota, J. I. Nieto-Hipolito, E. Jimenez-Garcia, and J. D. Sanchez-Lopez, "A proposal for using the internet of things concept to increase children's health awareness," in CONIELECOMP 2012, 22nd International Conference on Electrical Communications and Computers, pp. 168-172, Cholula, Puebla, Mexico, 2012.

[4] P. Pradeep, S. Krishnamoorthy, R. K. Pathinarupothi, and A. V. Vasilakos, "Leveraging context-awareness for Internet of Things ecosystem: representation, organization, and management of context," Computer Communications, vol. 177, pp. 33-50, 2021.

[5] Z. A. Almusaylim and N. Zaman, "A review on smart home present state and challenges: linked to context-awareness internet of things (IoT)," Wireless Networks, vol. 25, no. 6, pp. 3193-3204, 2019.

[6] J. Anesi, "Customer awareness, Internet of things influence IAQ products," Air Conditioning Heating \& Refrigeration, vol. 254, no. 8, pp. 42-44, 2015.

[7] G. L. Shi and Y. X. Liu, "Research on key technologies for Internet of things," Applied Mechanics \& Materials, vol. 190191, pp. 308-312, 2012.

[8] M. A. Albreem, A. M. Sheikh, M. H. Alsharif, M. Jusoh, and M. N. Mohd Yasin, "Green Internet of things (GIoT): applications, practices, awareness, and challenges," Access, vol. 9, pp. 38833-38858, 2021.

[9] Y. Yuan and D. Wang, "Path selection model and algorithm for emergency logistics management," Computers \& Industrial Engineering, vol. 56, no. 3, pp. 1081-1094, 2009.

[10] A. V. Donati, R. Montemanni, L. M. Gambardella, and A. E. Rizzoli, "Introduction to advanced system-on-chip test design and optimization, Erik Larson, Springer, Dordrecht (2005), 388 pp., Hardcover, plus XVIII, ISBN: 1-4020-3207-2," Microelectronics Reliability, vol. 48, no. 5, pp. 800-801, 2008.

[11] S. Behzadi, A. A. Alesheikh, and E. Poorazizi, "Developing a genetic algorithm to solve shortest path problem on a raster data Model," Journal of Applied Sciences, vol. 8, no. 18, pp. 3289-3293, 2008.

[12] Y. Zhang, J. H. Park, and K. T. Chong, "Model algorithm control for path tracking of wheeled mobile robots," International Journal of Precision Engineering and Manufacturing, vol. 11, no. 5, pp. 705-714, 2010.

[13] D. Q. Zhu, B. Sun, and L. Li, "Algorithm for AUV's 3-D path planning and safe obstacle avoidance based on biological inspired model," Control \& Decision, vol. 30, no. 5, pp. 798806, 2015.

[14] J. Dreo and P. Siarry, "Continuous interacting ant colony algorithm based on dense heterarchy," Future Generation Computer Systems, vol. 20, no. 5, pp. 841-856, 2004.

[15] G. L. Qin, “An improved ant colony algorithm based on adaptivelyadjusting pheromone," Information and Control, vol. 31, no. 3, 2002.

[16] L. Chen, J. Shen, L. Qin, and H. J. Chen, "An adaptive ant colony algorithm based on equilibrium of distribution," Journal of Software, vol. 14, no. 8, pp. 1379-1387, 2003.

[17] N. S. Safuan, M. Fathullah, Z. Shayfull, S. M. Nasir, and M. H. M. Hazwan, "Warpage improvement on wheel caster by opti- mizing the process parameters using genetic algorithm (GA)," AIP Conference Proceedings, vol. 1885, no. 1, pp. 1-5, 2017.

[18] T. A. Przylibski, "Radon and its daughter products behaviour in the air of an underground tourist route in the former arsenic and gold mine in Złoty Stok (Sudety Mountains, SW Poland)," Journal of Environmental Radioactivity, vol. 57, no. 2, pp. 87103, 2001.

[19] T. Przylibski, "Changes in the concentration of radon-222 and its daughter products in the air of the underground tourist route in Walim (Lower Silesia)," Antimicrobial Agents \& Chemotherapy, vol. 41, no. 3, pp. 196-205, 2000.

[20] D. E. Tchorz-Trzeciakiewicz and T. Parkitny, "Radon as a tracer of daily, seasonal and spatial air movements in the underground tourist route "Coal Mine" (SW Poland)," Journal of Environmental Radioactivity, vol. 149, pp. 90-98, 2015.

[21] Q. A. Huang, L. Dong, and L. F. Wang, " $L C$ passive wireless sensors toward a wireless sensing platform: status, prospects, and challenges," Journal of Microelectromechanical Systems, vol. 25, no. 5, pp. 822-841, 2016.

[22] C. Mandel, B. Kubina, M. Schüßler, and R. Jakoby, "Metamaterial-inspired passive chipless radio-frequency identification and wireless sensing," Annals of Telecommunications, vol. 68, no. 7-8, pp. 385-399, 2013.

[23] S. M. Loo, M. Owen, J. Kiepert, A. Planting, C. Spicer, and S. W. Dean, "Modular, portable, reconfigurable, and wireless sensing system for the aircraft cabin," Journal of ASTM International, vol. 5, no. 4, p. 101638, 2008.

[24] Z. Zhang, S. Schwartz, L. Wagner, and W. Miller, "A greedy algorithm for aligning DNA sequences," Journal of Computational Biology, vol. 7, no. 1-2, pp. 203-214, 2000.

[25] P. Hadikhani, M. Eslaminejad, M. Yari, and E. Ashoor Mahani, "An energy-aware and load balanced distributed geographic routing algorithm for wireless sensor networks with dynamic hole," Wireless Networks, vol. 26, no. 1, pp. 507519, 2020.

[26] S. Gu, G. Shi, W. Wu, and C. Lu, "A fast double greedy algorithm for non-monotone DR-submodular function maximization," Discrete Mathematics Algorithms and Applications, vol. 12, no. 1, 2020. 